\title{
Front Matter: Volume 7271
}

, "Front Matter: Volume 7271," Proc. SPIE 7271, Alternative Lithographic Technologies, 727101 (10 April 2009); doi: 10.1117/12.829689

SPIE. Event: SPIE Advanced Lithography, 2009, San Jose, California, United States 


\section{PROCEEDINGS OF SPIE}

\section{Alternative Lithographic Technologies}

Frank M. Schellenberg

Bruno M. La Fontaine

Editors

24-26 February 2009

San Jose, California, United States

Sponsored by

SPIE

Cooperating Organization

SEMATECH Inc.

Published by

SPIE 
The papers included in this volume were part of the technical conference cited on the cover and title page. Papers were selected and subject to review by the editors and conference program committee. Some conference presentations may not be available for publication. The papers published in these proceedings reflect the work and thoughts of the authors and are published herein as submitted. The publisher is not responsible for the validity of the information or for any outcomes resulting from reliance thereon.

Please use the following format to cite material from this book:

Author(s), "Title of Paper," in Alternative Lithographic Technologies, edited by Frank M. Schellenberg, Bruno M. La Fontaine, Proceedings of SPIE Vol. 7271 (SPIE, Bellingham, WA, 2009) Article CID Number.

ISSN 0277-786X

ISBN 9780819475244

Published by

SPIE

P.O. Box 10, Bellingham, Washington 98227-0010 USA

Telephone +1 3606763290 (Pacific Time) · Fax +1 3606471445

SPIE.org

Copyright (C) 2009, Society of Photo-Optical Instrumentation Engineers

Copying of material in this book for internal or personal use, or for the internal or personal use of specific clients, beyond the fair use provisions granted by the U.S. Copyright Law is authorized by SPIE subject to payment of copying fees. The Transactional Reporting Service base fee for this volume is $\$ 18.00$ per article (or portion thereof), which should be paid directly to the Copyright Clearance Center (CCC), 222 Rosewood Drive, Danvers, MA 01923. Payment may also be made electronically through CCC Online at copyright.com. Other copying for republication, resale, advertising or promotion, or any form of systematic or multiple reproduction of any material in this book is prohibited except with permission in writing from the publisher. The CCC fee code is 0277-786X/09/\$18.00.

Printed in the United States of America.

Publication of record for individual papers is online in the SPIE Digital Library.

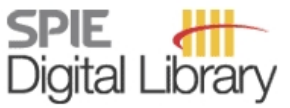

SPIEDigitalLibrary.org

Paper Numbering: Proceedings of SPIE follow an e-First publication model, with papers published first online and then in print and on CD-ROM. Papers are published as they are submitted and meet publication criteria. A unique, consistent, permanent citation identifier (CID) number is assigned to each article at the time of the first publication. Utilization of CIDs allows articles to be fully citable as soon they are published online, and connects the same identifier to all online, print, and electronic versions of the publication. SPIE uses a six-digit CID article numbering system in which:

- The first four digits correspond to the SPIE volume number.

- The last two digits indicate publication order within the volume using a Base 36 numbering system employing both numerals and letters. These two-number sets start with $00,01,02,03,04$, $05,06,07,08,09,0 A, 0 B \ldots 0 Z$, followed by 10-1Z, 20-2Z, etc.

The CID number appears on each page of the manuscript. The complete citation is used on the first page, and an abbreviated version on subsequent pages. Numbers in the index correspond to the last two digits of the six-digit CID number. 


\title{
Contents
}

\section{Part One}

\author{
xvii Symposium Committees \\ xix Conference Committee \\ xxiii Introduction
}

\section{SESSION 1 KEYNOTES: EUV}

727102 EUVL system: moving towards production (Keynote Paper) [7271-01]

H. Meiling, N. Buzing, ASML Netherlands B.V. (Netherlands); K. Cummings, N. Harned, ASML (United States); B. Hultermans, R. de Jonge, ASML Netherlands B.V. (Netherlands); B. Kessels, ASML (United States); P. Kürz, Carl Zeiss SMT AG (Germany); S. Lok, ASML Netherlands B.V. (Netherlands); M. Lowisch, Carl Zeiss SMT AG (Germany); J. Mallman, ASML Netherlands B.V. (United States); B. Pierson, ASML (United States); C. Wagner, A. van Dijk, E. van Setten, ASML Netherlands B.V. (Netherlands); J. Zimmerman, ASML (United States)

727103 LPP source system development for HVM (Keynote Paper)[7271-02]

D. C. Brandt, I. V. Fomenkov, A. I. Ershov, W. N. Partlo, D. W. Myers, N. R. Böwering, N. R. Farrar, G. O. Vaschenko, O. V. Khodykin, A. N. Bykanov, J. R. Hoffman, C. P. Chrobak, S. N. Srivastava, I. Ahmad, C. Rajyaguru, D. J. Golich, D. A. Vidusek, S. De Dea, R. R. Hou, Cymer, Inc. (United States)

727104 Integration of EUV lithography in the fabrication of 22-nm node devices (Keynote Paper) [7271-03]

O. Wood, Advanced Micro Devices, Inc. (United States); C.-S. Koay, K. Petrillo, IBM Corp. (United States); H. Mizuno, Toshiba America Electronic Components, Inc. (United States); S. Raghunathan, Advanced Micro Devices, Inc. (United States); J. Arnold, D. Horak, M. Burkhardt, G. McIntyre, IBM Corp. (United States); Y. Deng, B. La Fontaine, U. Okoroanyanwu, Advanced Micro Devices, Inc. (United States); A. Tchikoulaeva, AMD Saxony LLC \& Co. KG (Germany); T. Wallow, Advanced Micro Devices, Inc. (United States); J. H.-C. Chen, M. Colburn, S. S.-C. Fan, B. S. Haran, Y. Yin, IBM Corp. (United States)

\section{SESSION 2 KEYNOTES: ALTERNATIVE LITHO TECHNOLOGIES}

727106 On the integration of memristors with CMOS using nanoimprint lithography (Keynote Paper) [7271-05]

Q. Xia, Hewlett-Packard Labs. (United States); W. M. Tong, Hewlett-Packard Labs. (United States) and Hewlett Packard Co. (United States); W. WU, J. J. Yang, X. Li, W. Robinett, Hewlett-Packard Labs. (United States); T. Cardinali, M. Cumbie, J. E. Ellenson, Hewlett-Packard Co. (United States); P. Kuekes, R. S. Williams, Hewlett-Packard Labs. (United States)

727107 REBL nanowriter: Reflective Electron Beam Lithography (Keynote Paper) [7271-06] P. Petric, C. Bevis, A. Brodie, A. Carroll, A. Cheung, L. Grella, M. McCord, H. Percy, K. Standiford, M. Zywno, KLA-Tencor Corp. (United States) 
727108 Laser-produced plasma source development for EUV lithography [7271-07]

A. Endo, H. Komori, Y. Ueno, K. M. Nowak, Y. Takayuki, Y. Tatsuya, T. Suganuma, T. Asayama, H. Someya, H. Hoshino, M. Nakano, M. Moriya, T. Nishisaka, T. Abe, A. Sumitani, H. Nagano, Y. Sasaki, S. Nagai, Y. Watanabe, G. Soumagne, T. Ishihara, O. Wakabayashi, K. Kakizaki, H. Mizoguchi, EUVA (Japan)

727109 Xenon DPP source technologies for EUVL exposure tools [7271-08] M. Yoshioka, D. Bolshukhin, XTREME technologies GmbH (Germany); M. Corthout, Philips Extreme UV GmbH (Germany); G. H. Derra, Philips Research (Germany); S. Göłze, XTREME technologies GmbH (Germany); J. Jonkers, Philips Extreme UV GmbH (Germany); J. Kleinschmidt, R. Müller, M. C. Schürmann, G. Schriever, XTREME technologies GmbH (Germany); R. Snijkers, P. Zink, Philips Extreme UV GmbH (Germany)

7271 OC Design and fabrication considerations of EUVL collectors for HVM [7271-11] G. Bianucci, G. L. Cassol, J. Kools, M. Prea, G. Salmaso, G. Valsecchi, F. E. Zocchi, Media Lario Technologies (Italy); D. Bolshukhin, M. Schürmann, G. Schriever, XTREME technologies GmbH (Germany); A. Mader, P. Zink, Philips Extreme UV GmbH (Germany)

\section{SESSION 4 EUV MASK}

7271 OD Characteristics and issues of an EUVL mask applying phase-shifting thinner absorber for device fabrication [7271-12] H.-S. Seo, D.-G. Lee, B.-S. Ahn, H. Han, S. Huh, I.-Y. Kang, H. Kim, D. Kim, S.-S. Kim, H.-K. Cho, SAMSUNG Electronics Co., Ltd. (Korea, Republic of)

7271 OF Nanopit smoothing by cleaning [7271-14]

A. Rastegar, S. Eichenlaub, A. J. Kadaksham, M. House, SEMATECH, Inc. (United States)

7271 OG Compensation of overlay errors due to mask bending and non-flatness for EUV masks [7271-15]

M. Chandhok, S. Goyal, S. Carson, S.-J. Park, G. Zhang, Intel Corp. (United States); A. M. Myers, M. L. Leeson, Intel Corp. (Belgium); M. Kamna, F. C. Martinez, Intel Corp. (United States); A. R. Stivers, Consultant (United States); G. F. Lorusso, J. Hermans, E. Hendrickx, IMEC (Belgium); S. Govindjee, G. Brandstetter, Univ. of California, Berkeley (United States); T. Laursen, Duke Univ. (United States)

$7271 \mathrm{OH}$ Analysis of Coulomb and Johnsen-Rahbek electrostatic chuck performance in the presence of particles for EUV lithography [7271-17]

M. R. Sogard, Nikon Research Corp. of America (United States); A. R. Mikkelson, V. Ramaswamy, R. L. Engelstad, Univ. of Wisconsin, Madison (United States)

7271 ol Protection efficiency of a standard compliant EUV reticle handling solution [7271-18] L. He, SEMATECH, Inc. (United States) and Intel Corp. (United States); J. Lystad, Entegris, Inc. (United States); S. Wurm, K. Orvek, J. Sohn, SEMATECH, Inc. (United States); A. Ma, Intel Corp. (United States); P. Kearney, SEMATECH, Inc. (United States); S. Kolbow, D. Halbmaier, Entegris, Inc. (United States) 
7271 OK Cell projection use in maskless lithography for 45nm and 32nm logic nodes [7271-20]

S. Manakli, STMicroelectronics (France) and CEA-LETI, Minatec (France); H. Komami, M. Takizawa, ADVANTEST (Japan); T. Mitsuhashi, D2S, Inc. (Japan); L. Pain, CEA-LETI, Minatec (France)

$7271 \mathrm{OL}$ Optimal character-size exploration for increasing throughput of MCC lithographic systems [7271-21]

M. Sugihara, Toyohashi Univ. of Technology (Japan)

\section{SESSION 6 MULTIBEAM AND TOOLS PATTERNING}

$72710 N \quad$ PML2: the maskless multibeam solution for the 22nm node and beyond (Invited Paper) [7271-23]

C. Klein, E. Platzgummer, J. Klikovits, W. Piller, H. Loeschner, IMS Nanofabrication AG (Austria); T. Bejdak, P. Dolezel, V. Kolarik, DELONG INSTRUMENTS (Czech Republic);

W. Klingler, F. Letzkus, J. Butschke, M. Irmscher, Institut für Mikroelektronik Stuttgart (Germany); M. Witt, W. Pilz, Fraunhofer-Institut für Siliziumtechnologie (Germany);

P. Jaschinsky, Fraunhofer-Ctr. Nanoelectronic Technology (Germany); F. Thrum, C. Hohle, J. Kretz, Qimonda Dresden GmbH \& Co. OHG (Germany); J. T. Nogatch, A. Zepka, Synopsys, Inc. (United States)

$727100 \quad$ MAPPER: high-throughput maskless lithography [7271-24]

M. J. Wieland, G. de Boer, G. F. ten Berge, R. Jager, T. van de Peut, J. J. M. Peijster, E. Slot, S. W. H. K. Steenbrink, T. F. Teepen, A. H. V. van Veen, B. J. Kampherbeek, MAPPER Lithography B. V. (Netherlands)

$72710 Q \quad$ Coulomb blur advantage of a multi-shaped beam lithography approach [7271-26] M. Slodowski, H.-J. Doering, T. Elster, I. A. Stolberg, Vistec Electron Beam GmbH (Germany)

7271 OR Development of resist process for 5-KV multi-beam technology [7271-27]

B. Icard, D. Rio, CEA-LETI, Minatec (France); P. Veltman, B. Kampherbeek, MAPPER Lithography B.V. (Netherlands); C. Constancias, L. Pain, CEA-LETI, Minatec (France)

\section{SESSION 7 EUV PRINTING}

7271 OT Stability and imaging of the ASML EUV alpha demo tool [7271-29]

J. V. Hermans, B. Baudemprez, G. Lorusso, E. Hendrickx, IMEC (Belgium); A. van Dijk, ASML Netherlands B.V. (Netherlands); R. Jonckheere, A.-M. Goethals, IMEC (Belgium)

$72710 U$ Flare evaluation of ASML alpha demo tool [7271-30]

H. Mizuno, Toshiba America Electronic Components, Inc. (United States); G. Mclntyre, C. Koay, M. Burkhardt, IBM Corp. (United States); B. La Fontaine, O. Wood, Advanced Micro Devices, Inc. (United States) 
7271 OW The SEMATECH Berkeley microfield exposure tool: learning at the 22-nm node and beyond [7271-32]

P. P. Naulleau, Lawrence Berkeley National Lab. (United States); C. N. Anderson, Univ. of California, Berkeley (United States); L.-M. Baclea-an, P. Denham, S. George, K. A. Goldberg, Lawrence Berkeley National Lab. (United States); M. Goldstein, SEMATECH, Inc. (United States); B. Hoef, Lawrence Berkeley National Lab. (United States); R. Hudyma, Hyperion (United States); G. Jones, Lawrence Berkeley National Lab. (United States); C. Koh, SEMATECH, Inc. (United States); B. La Fontaine, Advanced Micro Devices, Inc. (United States); B. McClinton, R. Miyakawa, Lawrence Berkeley National Lab. (United States); W. Montgomery, SEMATECH, Inc. (United States); J. Roller, Lawrence Berkeley National Lab. (United States); T. Wallow, Advanced Micro Devices, Inc. (United States); S. Wurm, SEMATECH, Inc. (United States)

7271 OX Out-of-band exposure characterization with the SEMATECH Berkeley 0.3-NA microfield exposure tool [7271-33]

S. A. George, P. P. Naulleau, S. Rekawa, E. Gullikson, C. D. Kemp, Lawrence Berkeley National Lab. (United States)

7271 OY Estimation of cost comparison of lithography technologies at the 22-nm half-pitch node [7271-34] A. Wüest, SEMATECH, Inc. (United States); A. J. Hazelton, Nikon Corp. (Japan); G. Hughes, SEMATECH, Inc. (United States)

\section{SESSION 8 EUV LIFETIME}

727110 Carbon film growth on model electron-irradiated MLM cap layer: interaction of benzene and MMA vapor with $\mathrm{TiO}_{2}$ surface [7271-36]

B. V. Yakshinskiy, S. Zalkind, R. A. Bartynski, Rutgers Univ. (United States); R. Caudillo, Intel Corp. (United States); T. E. Madey, Rutgers Univ. (United States)

727112 Tracking down sources of carbon contamination in EUVL exposure tools [7271-38] C. Tarrio, R. E. Vest, T. B. Lucatorto, National Institute of Standards and Technology (United States); R. Caudillo, Intel Corp. (United States)

727113 Measuring the EUV-induced contamination rates of $\mathrm{TiO}_{2}$-capped multilayer optics by anticipated production-environment hydrocarbons [7271-39]

S. B. Hill, National Institute of Standards and Technology (United States); N. S. Faradzhev, Rutgers Univ. (United States); C. S. Tarrio, T. B. Lucatorto, National Institute of Standards and Technology (United States); R. A. Bartynski, B. V. Yakshinskiy, T. E. Madey, Rutgers Univ. (United States)

\section{SESSION 9 EUV APPLICATIONS}

727114 The application of EUV lithography for 40nm node DRAM device and beyond [7271-40] J. Park, C. Koh, D. Goo, I. Kim, C. Park, J. Lee, J. Park, J. Yeo, S.-W. Choi, C. Park, SAMSUNG Electronics Co., Ltd. (Korea, Republic of) 
727115 Comparative study of DRAM cell patterning between ArF immersion and EUV lithography [7271-41]

T.-S. Eom, S. Park, J.-T. Park, C.-M. Lim, S. Koo, Y.-S. Hyun, H. Kim, B.-H. Nam, C.-R. Kim,

S.-C. Moon, N.-J. Kwak, S. Park, Hynix Semiconductor Inc. (Korea, Republic of)

727116 Demonstration of full-field patterning of $\mathbf{3 2} \mathbf{~ n m}$ test chips using EUVL [7271-42]

G. Vandentop, M. Chandhok, E. S. Putna, T. R. Younkin, J. S. Clarke, S. Carson, Intel Corp. (United States); A. Myers, M. Leeson, Intel Corp. (Belgium); G. Zhang, T. Liang, Intel Corp. (United States); T. Murachi, Intel Corp. (Japan)

727117 EUVL reticle defectivity evaluation [7271-43]

A. Tchikoulaeva, AMD Saxony LLC \& Co. KG (Germany); U. Okoroanyanwu, O. Wood, B. La Fontaine, Advanced Micro Devices, Inc. (United States); C. Holfeld, Advanced Mask Technology Ctr. (Germany); S. Kini, KLA-Tencor Corp. (United States); M. Peikert, KLA Tencor GmbH (Germany) C. Boye, C. Koay, K. Petrillo, IBM Corp. (United States); H. Mizuno, Toshiba America Electronic Components, Inc. (United States)

727118 Recent progress of EUV full-field exposure tool in Selete [7271-44]

K. Tawarayama, H. Aoyama, S. Magoshi, Y. Tanaka, S. Shirai, H. Tanaka, Semiconductor Leading Edge Technologies, Inc. (Japan)

SESSION 10 EUV OPC

727119 Exposure tool settings and OPC strategies for EUV lithography at the 16-nm node [7271-45] Y. Deng, J. Kye, B. La Fontaine, T. Wallow, O. Wood, H. Levinson, GLOBALFOUNDRIES Inc. (United States); A. Fumar-Pici, H. Mizuno, Toshiba America Electronic Components, Inc. (United States); C. Koay, G. McIntyre, IBM Corp. (United States)

7271 1A Requirements and results of a full-field EUV OPC flow [7271-46]

S. Jang, L. Zavyalova, B. Ward, K. Lucas, Synopsys, Inc. (United States)

7271 1B Imaging budgets for EUV optics: ready for 22-nm node and beyond [7271-47]

M. Bienert, A. Göhnemeier, O. Natt, M. Lowisch, P. Gräupner, T. Heil, R. Garreis, Carl Zeiss SMT AG (Germany); K. van Ingen Schenau, ASML Netherlands B.V. (Netherlands); S. Hansen, ASML (United States)

7271 1C Modeling and experiments of non-telecentric thick mask effects for EUV lithography [7271-48]

G. Mclntyre, C. Koay, M. Burkhardt, IBM Corp. (United States); H. Mizuno, Toshiba America,

Inc. (United States); O. Wood, Advanced Micro Devices, Inc. (United States)

7271 1E Mask diffraction analysis and optimization for EUV masks [7271-144]

A. Erdmann, P. Evanschitzky, T. Fühner, Fraunhofer Institute of Integrated Systems and Device Technology (Germany)

7271 IF Comparison of fast 3D simulation and actinic inspection for EUV masks with buried defects [7271-50]

C. H. Clifford, S. Wiraatmadja, T. T. Chan, A. R. Neureuther, Univ. of California, Berkeley (United States); K. A. Goldberg, I. Mochi, Lawrence Berkeley National Lab. (United States); T. Liang, Intel Corp. (United States) 
7271 1G EUV-patterning characterization using a 3D mask simulation and field EUV scanner $[7271-51]$

J.-T. Park, Y.-S. Hyun, C.-M. Lim, T.-S. Eom, S. Koo, S. Park, S.-K. Kim, K.-D. Ban, H.-J. Yang,

C.-I. Oh, B.-H. Nam, C.-R. Kim, H. Kim, S.-C. Moon, S. Park, Hynix Semiconductor Inc. (Korea, Republic of)

\section{SESSION 11 MASKLESS}

$7271 \mathrm{lH} \quad$ Full-chip characterization of compression algorithms for direct-write maskless lithography systems (Invited Paper) [7271-52]

A. Zakhor, V. Dai, G. Cramer, Univ. of California, Berkeley (United States)

$727111 \quad$ Scalable (24-140 Gbps) optical data link well adapted for future maskless lithography applications [7271-53]

A. Paraskevopoulos, S.-H. Voss, M. Talmi, G. Walf, Fraunhofer-Institut for Telecommunications, Heinrich-Hertz-Institut (Germany)

\section{SESSION 12 NANOIMPRINT I}

$7271 \mathrm{lL} \quad$ Step and flash imprint lithography for manufacturing patterned media (Invited Paper) [7271-56]

C. Brooks, G. M. Schmid, M. Miller, S. Johnson, N. Khusnatdinov, D. LaBrake, D. J. Resnick, S. V. Sreenivasan, Molecular Imprints, Inc. (United States)

$72711 \mathrm{M} \quad$ UV-NIL template making and imprint evaluation [7271-94]

S. Sasaki, T. Hiraka, J. Mizuochi, Y. Nakanishi, S. Yusa, Y. Morikawa, H. Mohri, N. Hayashi, Dai Nippon Printing Co., Ltd. (Japan)

727110 Soft stamp UV-nanoimprint lithography for fabrication of laser diodes [7271-58] J. Viheriälä, M.-R. Viljanen, J. Kontio, T. Leinonen, J. Tommila, M. Dumitrescu, T. Niemi, M. Pessa, Tampere Univ. of Technology (Finland)

\section{SESSION 13 NANOIMPRINT II}

$72711 Q \quad$ SEMATECH's nanolmprint program: a key enabler for nanoimprint introduction [7271-60] L. C. Litt, SEMATECH, Inc. (United States) and Advanced Micro Devices, Inc. (United States); M. Malloy, SEMATECH, Inc. (United States)

7271 is Direct laser write (DLW) as a versatile tool in manufacturing templates for imprint lithography on flexible substrates [7271-62]

M. G. Ivan, J.-B. Vaney, TNO Science and Industry (Netherlands); D. Verhaart, Singulus Mastering B.V. (Netherlands); E. R. Meinders, TNO Science and Industry (Netherlands)

$72711 \mathrm{E}$ Evaluation of the CD-SEM Vistec LWM90xx for line-width measurement of nanoimprint templates [7271-64]

M. Pritschow, J. Butschke, M. Irmscher, Institut für Mikroelektronik Stuttgart (Germany); L. Parisoli, KLA-Tencor Corp. (Germany); T. Oba, T. Iwai, T. Nakamura, Advantest Corp. (Japan) 
7271 IV Physical properties of thin nanoimprint polymer films measured by photo-acoustic metrology [7271-65]

T. Kehoe, Univ. Autonoma de Barcelona (Spain) and Univ. College Cork (Ireland); J. Bryner, ETH Zürich (Switzerland); V. Reboud, Univ. Autonoma de Barcelona (Spain); J. Vollmann, ETH Zürich (USA); C. M. Sotomayor Torres, Univ. Autonoma de Barcelona (Spain) and Institute for Research and Advanced Studies (Spain)

7271 IW High-resolution defect inspection of step-and-flash imprint lithography for 32-nm half-pitch patterning [7271-66]

K. Selinidis, E. Thompson, I. McMackin, S. Sreenivasan, D. J. Resnick, Molecular Imprints, Inc. (United States)

\section{SESSION 14 EUV TOOLS}

7271 1X Nikon EUVL development progress update [7271-67]

T. Miura, K. Murakami, H. Kawai, Y. Kohama, K. Morita, K. Hada, Y. Ohkubo, Nikon Corp. (Japan)

7271 IY Development status of Canon's full-field EUVL tool [7271-68]

T. Hasegawa, S. Uzawa, T. Honda, Y. Higaki, A. Miyake, H. Morishima, Canon Inc. (Japan)

727112 Development progress of optics for EUVL at Nikon [7271-69]

K. Murakami, T. Oshino, H. Kondo, M. Shiraishi, H. Chiba, H. Komatsuda, K. Nomura,

J. Nishikawa, Nikon Corp. (Japan)

727120 Process liability evaluation for EUVL [7271-74]

H. Aoyama, K. Tawarayama, Y. Tanaka, D. Kawamura, Y. Arisawa, T. Uno, T. Kamo,

T. Tanaka, T. Itani, H. Tanaka, Semiconductor Leading Edge Technologies, Inc. (Japan);

Y. Nakajima, R. Inanami, K. Takai, K. Murano, T. Koshiba, K. Hashimoto, Toshiba Corp.

Semiconductor Co. (Japan); I. Mori, Semiconductor Leading Edge Technologies, Inc. (Japan)

727121 Characterization of a 0.25NA full-field EUV exposure tool [7271-70]

O. Kritsun, B. La Fontaine, GLOBALFOUNDRIES Inc. (United States); Y. Hao, J. Li, Nanometrics Inc. (United States); O. Wood, S. Raghunathan, GLOBALFOUNDRIES Inc. (United States); T. Brunner, C.-S. Koay, IBM Corp. (United States); H. Mizuno, Toshiba America Electronics Components (United States)

727122 Development of actinic full-field EUV mask blank inspection tool at MIRAI-Selete [7271-71] T. Terasawa, T. Yamane, T. Tanaka, T. Iwasaki, O. Suga, Semiconductor Leading Edge Technologies, Inc. (Japan); T. Tomie, National Institute of Advanced Industrial Science and Technology (Japan)

727123 Improving the performance of the actinic inspection tool with an optimized alignment procedure [7271-72]

I. Mochi, K. A. Goldberg, P. Naulleau, Lawrence Berkeley National Lab. (United States);

S. Huh, SEMATECH, Inc. (United States) 


\section{Part Two}

\section{SESSION 15 EUV RESIST}

727124 Assessment of EUV resist readiness for 32-nm hp manufacturing and extendibility study of EUV ADT using state-of-the-art resist [7271-73]

C. Koh, L. Ren, J. Georger, F. Goodwin, S. Wurm, SEMATECH, Inc. (United States); B. Pierson, ASML US, Inc. (United States); J. Park, SAMSUNG Electronics Co., Ltd. (Korea, Republic of); T. Wallow, Advanced Micro Devices, Inc. (United States); T. R. Younkin, Intel Corp. (United

States); P. Naulleau, Lawrence Berkeley National Lab. (United States)

727126 EUV resist outgassing: scaling to HVM intensity [7271-76]

A. O. Antohe, C. Mbanaso, Y.-J. Fan, L. Yankulin, R. Garg, P. Thomas, G. Denbeaux, Univ. at Albany (United States); E. C. Piscani, A. F. Wuest, SEMATECH, Inc. (United States)

\section{SESSION 16 NANOIMPRINT MATERIALS}

727128 In situ measurement of annealing-induced line shape evolution in nanoimprinted polymers using scatterometry [7271-79]

H. J. Patrick, National Institute of Standards and Technology (United States) and KT Consulting, Inc. (United States); T. A. Germer, National Institute of Standards and Technology (United States); Y. Ding, Univ. of Colorado, Boulder (United States); H. W. Ro, L. J. Richter, C. L. Soles, National Institute of Standards and Technology (United States)

727129 Characterization of vinyl ether UV-cure nanoimprint resist [7271-80] T. Furukawa, JSR Micro, Inc. (United States); F. A. Houle, D. L. Casher, D. C. Miller, IBM Almaden Research Ctr. (United States)

7271 2A A study of filling process for UV nanoimprint lithography using a fluid simulation [7271-81] I. Yoneda, Semiconductor Co. Toshiba Corp. (Japan); Y. Nakagawa, Toshiba Corp. (Japan); S. Mikami, H. Tokue, T. Ota, T. Koshiba, M. Ito, K. Hashimoto, T. Nakasugi, T. Higashiki, Semiconductor Co., Toshiba Corp. (Japan)

\section{SESSION 17 DIRECTED SELF ASSEMBLY}

$72712 \mathrm{D} \quad$ Lithographically directed materials assembly [7271-84]

R. P. Kingsborough, R. B. Goodman, K. Krohn, T. H. Fedynyshyn, MIT Lincoln Lab. (United States)

\section{NANOIMPRINT: POSTER SESSION}

$72712 \mathrm{H} \quad$ Automated imprint mask cleaning for step-and-flash imprint lithography [7271-88] S. Singh, S. Chen, HamaTech APE Inc. (United States); K. Selinidis, B. Fletcher, I. McMackin, E. Thompson, D. J. Resnick, Molecular Imprints, Inc. (United States); P. Dress, HamaTech APE GmbH \& Co. KG (Germany); U. Dietze, HamaTech APE Inc. (United States) 
727121 Optimization of droplets for UV-NIL using coarse-grain simulation of resist flow [7271-89] V. Sirotkin, A. Svintsov, S. Zaitsev, Institute of Microelectronics Technology (Russian Federation)

$72712 \mathrm{~J}$ Industrial applications demanding low and high resolution features realized by soft UV-NIL and hot embossing [7271-90]

R. Miller, EVGroup Inc. (United States); T. Glinsner, G. Kreindl, P. Lindner, M. Wimplinger, EV Group Inc. (Austria)

\section{E-BEAM AND MASKLESS: POSTER SESSION}

$72712 \mathrm{M} \quad$ Fabrication of metrology test structures for future technology nodes using high-resolution variable-shaped e-beam direct write [7271-95]

L. Szikszai, P. Jaschinsky, K. Keil, M. Hauptmann, Fraunhofer Ctr. Nanoelectronic Technologies (Germany); M. Mört, U. Seifert, C. Hohle, K.-H. Choi, F. Thrum, J. Kretz, Qimonda Dresden GmbH \& Co. OHG (Germany); V. Ferreras Paz, Univ. of Stuttgart (Germany); A. den Boef, ASML Netherlands B.V. (Netherlands)

$72712 \mathrm{~N}$ Sub-30-nm resolution parallel EB lithography based on a planar type Si nanowire array ballistic electron source [7271-96]

A. Kojima, Crestec Corp. (Japan) and Tokyo Univ. of Agriculture and Technology (Japan); H. Ohyi, Crestec Corp. (Japan); N. Koshida, Tokyo Univ. of Agriculture and Technology (Japan)

727120 Optimization of BSE-detector for e-beam direct write lithography [7271-97]

H. Alves, P. Hahmann, M. Slodowski, Vistec Electron Beam GmbH (Germany); C. G. Frase,

D. Gnieser, K.-P. Johnsen, H. Bosse, Physikalisch-Technische Bundesanstalt (Germany)

7271 2P 3D ion multibeam processing with the CHARPAN PMLP tool and with the single ion-beam FIB tool optimized with the lonRevSim software [7271-98]

S. Zaitsev, A. Svintsov, Institute of Microelectronics Technology and High Purity Materials (Russian Federation); C. Ebm, S. Eder-Kapl, H. Loeschner, E. Platzgummer, IMS Nanofabrication AG (Austria); G. Lalev, S. Dimov, V. Velkova, Cardiff Univ. (United Kingdom); B. Basnar, Vienna Univ. of Technology (Austria)

7271 2R Monitor and self-diagnostic technology for mask e-beam writing system [7271-100] N. Samoto, H. Manabe, O. Wakimoto, S. lida, H. Hoshi, M. Yamabe, Association of Super-Advanced Electronics Technologies (Japan)

7271 2S Proximity effect correction for 20nm dimension patterning [7271-101]

D. Tsunoda, M. Shoji, M. Tatsugawa, H. Tsunoe, Y. lino, Nippon Control System Corp. (Japan); P. Jedrasik, Chalmers Univ. of Technology (Sweden)

\section{NOVEL TECHNOLOGIES: POSTER SESSION}

$72712 T \quad$ Fabrication of sub-10-nm pattern using diblock copolymer [7271-102]

N. Kihara, K. Takizawa, H. Hieda, Toshiba Corp. (Japan) 
$72712 \mathrm{U} \quad$ Interference assisted lithography for patterning of 1D gridded design [7271-103]

R. T. Greenway, Petersen Advanced Lithography, Inc. (United States); R. Hendel, Applied Materials, Inc. (United States); K. Jeong, A. B. Kahng, Univ. of California, San Diego (United States); J. S. Petersen, Petersen Advanced Lithography, Inc. (United States); Z. Rao, Applied Materials, Inc. (United States); M. C. Smayling, Tela Innovations, Inc. (United States)

\section{EUV SOURCE: POSTER SESSION}

7271 2W Kinetic simulation of debris from an LPP EUV source [7271-105]

B. Rollinger, A. Giovannini, D. Bleiner, N. Chokani, R. S. Abhari, ETH Zürich (Switzerland)

7271 2X High-performance next-generation EUV lithography light source [7271-106]

P. Choi, NANO-UV SAS (France) and EPPRA SAS (France); S. V. Zakharov, EPPRA SAS (France) and RRC Kurchatov Institute (Russian Federation); R. Aliaga-Rossel, NANO-UV SAS (France); O. Benali, EPPRA SAS (France); G. Duffy, NANO-UV SAS (France); O. Sarroukh, EPPRA SAS (France); E. Wyndham, Pontificia Univ. Catolica de Chile (Chile); V. S. Zakharov, EPPRA SAS (France), RRC Kurchatov Institute (Russian Federation), and Keldysh Institute of Applied Mathematics (Russian Federation)

$72712 Y \quad$ Grid spectral purity filters for suppression of infrared radiation in laser-produced plasma EUV sources [7271-108] W. A. Soer, M. J. J. Jak, Philips Research (Netherlands); A. M. Yakunin, ASML (Netherlands); M. M. J. W. van Herpen, Philips Research (Netherlands); V. Y. Banine, ASML (Netherlands)

$72712 Z$ Ablation depth in planar Sn targets during the interaction with a Nd:YAG laser for extreme ultraviolet lithography [7271-109]

R. A. Burdt, S. Yuspeh, K. L. Sequoia, M. S. Tillack, Y. Tao, F. Najmabadi, Ctr. for Energy Research (United States)

727130 Atomic processes in the LPP and LA-DPP EUV sources [7271-110] A. Sasaki, Japan Atomic Energy Agency (Japan); K. Nishihara, A. Sunahara, H. Furukawa, Osaka Univ. (Japan); T. Nishikawa, Okayama Univ. (Japan); F. Koike, Kitasato Univ. (Japan)

727131 Remote plasma cleaning of Sn from an EUV collector mirror [7271-112] H. Shin, R. Raju, D. N. Ruzic, Univ. of Illinois at Urbana-Champaign (United States)

727132 Debris characteristics and mitigation of a laser plasma tin-contained liquid jet/droplet targets [7271-113]

M. Kaku, S. Touge, M. Katto, S. Kubodera, Univ. of Miyazaki (Japan)

727133 Evaluation at the intermediate focus for EUV light source [7271-114] T. Suganuma, G. Soumagne, M. Moriya, T. Abe, A. Sumitani, A. Endo, EUVA (Japan)

727135 Performance evaluation of source collector module for extreme ultraviolet small-field exposure tool [7271-116]

S. Magoshi, S. Shirai, H. Mori, K. Tawarayama, Y. Tanaka, H. Tanaka, Semiconductor Leading Edge Technologies, Inc. (Japan) 
727136 Effects of the ratio of sphere size to laser focal spot on the dominant in-band EUV emitting region [7271-117]

S. Yuspeh, K. L. Sequoia, Y. Tao, M. S. Tillack, R. A. Burdt, F. Najmabadi, Univ. of California, San Diego (United States) and Ctr. for Energy Research (United States)

727137 Measurement of particle flux at the intermediate focus of a DPP source [7271-118] J. Sporre, R. Raju, D. N. Ruzic, V. Surla, Univ. of Illinois at Urbana-Champaign (United States); F. Goodwin, SEMATECH, Inc. (United States)

727138 Laser-produced plasma light source for EUVL [7271-119] I. V. Fomenkov, D. C. Brandt, A. N. Bykanov, A. I. Ershov, W. N. Partlo, D. W. Myers, N. R. Böwering, N. R. Farrar, G. O. Vaschenko, O. V. Khodykin, J. R. Hoffman, C. P. Chrobak, S. N. Srivastava, D. J. Golich, D. A. Vidusek, S. De Dea, R. R. Hou, Cymer, Inc. (United States)

727139 Dependence of laser parameter on conversion efficiency in high-repetition-rate laser-ablation-discharge EUV source [7271-120]

Y. Teramoto, T. Yokoyama, H. Mizokoshi, H. Sato, K. Hotta, EUVA (Japan)

$72713 A$ Development of a high-pulse-rate EUV source [7271-121]

S. F. Horne, F. M. Niell, M. J. Partlow, M. M. Besen, D. K. Smith, P. A. Blackborow, D. Gustafson, Energetiq Technology, Inc. (United States)

7271 3B Optical element for full spectral purity from IR-generated EUV light sources [7271-159] A. J. R. van den Boogaard, E. Louis, FOM Institute for Plasma Physics Rijnhuizen (Netherlands); F. A. van Goor, Univ. Twente (Netherlands); F. Bijkerk, FOM Institute for Plasma Physics Rijnhuizen (Netherlands) and Univ. of Twente (Netherlands)

\section{EUV MASK: POSTER SESSION}

7271 3C Removal of contaminants by plasma assisted cleaning by metastable atom neutralization (PACMAN) [7271-122]

W. M. Lytle, D. S. Szybilski, C. E. Das, R. Raju, D. N. Ruzic, Univ. of Illinois at Urbana-Champaign (United States)

$72713 \mathrm{D}$ Precise evaluation of zero-CTE temperature of EUVL-grade $\mathrm{TiO}_{2}-\mathrm{SiO}_{2}$ Ultra-low-expansion glass using the line-focus-beam ultrasonic material characterization system [7271-123] J. Kushibiki, M. Arakawa, Tohoku Univ. (Japan)

$72713 F \quad$ Inspection 13.2-nm table-top full-field microscope [7271-125]

F. Brizuela, Y. Wang, C. A. Brewer, F. Pedaci, Colorado State Univ., Fort Collins (United States); W. Chao, E. H. Anderson, Y. Liu, K. A. Goldberg, P. P. Naulleau, Lawrence Berkeley National Lab. (United States); P. W. Wachulak, M. C. Marconi, Colorado State Univ., Fort Collins (United States); D. T. Attwood, Lawrence Berkeley National Lab. (United States); J. J. Rocca, C. S. Menoni, Colorado State Univ., Fort Collins (United States)

$72713 G \quad$ Experimental study of particle-free mask handling [7271-126] M. Amemiya, K. Ota, T. Taguchi, O. Suga, Semiconductor Leading Edge Technologies, Inc. (Japan) 
$72713 \mathrm{H}$ The performance of an actinic full-field EUVL mask blank inspection system [7271-127] T. Yamane, T. Iwasaki, T. Tanaka, T. Terasawa, O. Suga, Semiconductor Leading Edge Technologies, Inc. (Japan); T. Tomie, National Institute of Advanced Industrial Science and Technology (Japan)

$727131 \quad$ Defect printability of thin absorber mask in EUV lithography [7271-128] T. Kamo, H. Aoyama, Y. Arisawa, T. Tanaka, O. Suga, Semiconductor Leading Edge Technologies, Inc. (Japan)

7271 3J Mask defect verification using actinic inspection and defect mitigation technology [7271-129]

S. Huh, P. Kearney, S. Wurm, F. Goodwin, SEMATECH, Inc. (United States); K. Goldberg, I. Mochi, E. Gullikson, Lawrence Berkeley National Lab. (United States)

7271 3K Analysis of a relation between the spatial frequency of electrostatic chuck and induced mask inplane distortion (IPD) [7271-130]

T. Yamamoto, The Univ. of Tokyo (Japan); K. Ota, N. Nishimura, Semiconductor Leading Edge Technologies, Inc. (Japan); S. Warisawa, S. Ishihara, The Univ. of Tokyo (Japan)

7271 3L Characterization of electrostatic chucks for extreme ultraviolet lithography [7271-131] T. C. Mulholland, J. R. Zeuske, P. Vukkadala, R. L. Engelstad, Univ. of Wisconsin, Madison (United States)

$72713 \mathrm{M}$ Experimental evaluation of particulate contamination on backside of EUV reticle [7271-132] K. Ota, T. Taguchi, M. Amemiya, N. Nishimura, O. Suga, Semiconductor Leading Edge Technologies, Inc. (Japan)

$72713 \mathrm{~N} \quad$ Collecting EUV mask images through focus by wavelength funing [7271-155] K. A. Goldberg, I. Mochi, Lawrence Berkeley National Lab. (United States); S. Huh, SEMATECH Inc. (United States)

\section{EUV PERFORMANCE: POSTER SESSION}

727130 Extreme ultraviolet holographic lithography with a table-top laser [7271-133]

A. Isoyan, F. Jiang, Y.-C. Cheng, Univ. of Wisconsin, Madison (United States); P. Wachulak, L. Urbanski, J. Rocca, C. Menoni, M. Marconi, Colorado State Univ. (United States);

F. Cerrina, Univ. of Wisconsin, Madison (United States)

$72713 \mathrm{P}$ Analysis of carbon deposition on multilayer mirrors by using two different beamlines [7271-134]

T. Nakayama, A. Miyake, H. Takase, S. Terashima, T. Sudo, Y. Watanabe, Y. Fukuda, Canon Inc. (Japan)

$72713 Q \quad$ Assumptions and trade-offs of extreme ultraviolet optics contamination modeling [7271-135]

V. Jindal, SEMATECH, Inc. (United States); R. Garg, G. Denbeaux, Univ. at Albany (United States); A. Wüest, SEMATECH, Inc. (United States)

$72713 R$ Durability of capped multilayer mirrors for high volume manufacturing extreme ultraviolet lithography tool [7271-136]

S. Matsunari, Y. Kakutani, T. Aoki, S. Kawata, K. Murakami, Nikon Corp. (Japan) 
7271 3S Fabrication of half-pitch 32-45-nm SRAM patterns with EUVL [7271-137]

Y. Tanaka, H. Aoyama, S. Magoshi, K. Tawarayama, S. Shirai, H. Tanaka, Semiconductor

Leading Edge Technologies, Inc. (Japan)

$72713 \mathrm{U}$ Carbon contamination of extreme ultraviolet (EUV) masks and its effect on imaging [7271-139]

Y.-J. Fan, L. Yankulin, A. Antohe, R. Garg, P. Thomas, C. Mbanaso, Univ. at Albany (United

States); A. Wüest, F. Goodwin, S. Huh, SEMATECH, Inc. (United States); P. Naulleau,

K. Goldberg, I. Mochi, Lawrence Berkeley National Lab. (United States); G. Denbeaux, Univ. at Albany (United States)

$72713 \mathrm{~V}$ Improved contrast and reflectivity of multilayer reflective optics for wavelengths beyond the extreme UV [7271-156]

T. Tsarfati, E. Zoethout, E. Louis, R. van de Kruijs, A. Yakshin, FOM Institute for Plasma Physics Rijnhuizen (Netherlands); S. Müllender, Carl Zeiss SMT AG (Germany); F. Bijkerk, FOM Institute for Plasma Physics Rijnhuizen (Netherlands) and Univ. of Twente (Netherlands)

\section{EUV METROLOGY: POSTER SESSION}

$72713 X \quad$ EUVL dosimetry at NIST [7271-140]

C. Tarrio, S. Grantham, M. Cangemi, R. E. Vest, T. B. Lucatorto, National Institute of Standards and Technology (United States); N. Harned, ASML (United States)

7271 3Y High-accuracy EUV reflectometry at large optical components and oblique incidence [7271-141]

C. Laubis, F. Scholze, C. Buchholz, A. Fischer, S. Hesse, A. Kampe, J. Puls, C. Stadelhoff, G. Ulm, Physikalisch-Technische Bundesanstalt (Germany)

727140 Ellipsometric and surface acoustic wave sensing of carbon contamination on EUV optics [7271-157]

J. Chen, E. Louis, FOM Institute for Plasma Physics Rijnhuizen (Netherlands); F. Bijkerk, C. J. Lee, FOM Institute for Plasma Physics Rijnhuizen (Netherlands) and Univ. Twente (Netherlands); H. Wormeester, Univ. Twente (Netherlands); R. Kunze, H. Schmidt, Leibniz Institute for Solid State and Materials Research (Germany); D. Schneider, Fraunhofer Institute for Material and Beam Technology (Germany); R. Moors, W. van Schaik, M. Lubomska, ASML B.V. (Netherlands)

EUV OPC: POSTER SESSION

7271 41 Analysis of EUVL mask effects under partially coherent illumination [7271-145] V. Domnenko, Synopsys, Inc. (Russian Federation); T. Schmoeller, T. Klimpel, Synopsys GmbH (Germany)

727142 Assessment of full-chip level EUV optical correction for sub-40nm memory device [7271-146]

J. Lee, I. Kim, D. Goo, J. Park, C. Park, J. Park, J. Yeo, S. Choi, W. Han, SAMSUNG Electronics

Co., Ltd. (Korea, Republic of) 
727143 Incident angle change caused by different off-axis illumination in extreme ultraviolet lithography [7271-147]

E.-J. Kim, J.-H. You, Hanyang Univ. (Korea, Republic of); S.-S. Kim, H.-K. Cho, SAMSUNG

Electronics Co., Ltd. (Korea, Republic of); I. An, H.-K. Oh, Hanyang Univ. (Korea, Republic of)

727144 Evaluation of shadowing and flare effect for EUV tool [7271-149]

J. Moon, C.-K. Kim, B.-S. Nam, B.-H. Nam, Y.-S. Hyun, S.-K. Kim, C.-M. Lim, Y.-D. Kim, M.-S. Kim, Y.-K. Choi, C.-R. Kim, D. Yim, Hynix Semiconductor Inc. (Korea, Republic of)

727145 Flare compensation for EUVL [7271-150]

Y. Arisawa, H. Aoyama, T. Uno, T. Tanaka, I. Mori, Semiconductor Leading Edge

Technologies, Inc. (Japan)

\section{EUV RESIST: POSTER SESSION}

727146 Measurement and analysis of EUV photoresist related outgassing and contamination [7271-152]

I. Pollentier, IMEC (Belgium); G. Aksenov, Katholieke Univ. Leuven (Belgium); A.-M. Goethals, R. Gronheid, R. Jonckheere, IMEC (Belgium); M. Leeson, Intel Corp. (United States)

727147 RLS tradeoff vs. quantum yield of high PAG EUV resists [7271-153]

C. Higgins, A. Antohe, G. Denbeaux, S. Kruger, Univ. at Albany (United States); J. Georger, SEMATECH, Inc. (United States); R. Brainard, Univ. at Albany (United States)

727148 Investigation of EUV-process sensitivities for wafer-track processing [7271-154]

N. Bradon, H. Weichert, Tokyo Electron Europe Ltd. (United Kingdom); K. Nafus, S. Hatakeyama, J. Kitano, H. Kosugi, K. Yoshihara, Tokyo Electron Kyushu Ltd. (Japan); M. Goethals, J. Hermans, IMEC (Belgium)

Author Index 


\title{
Symposium Committees
}

\author{
Symposium Chair
}

Christopher J. Progler, Photronics, Inc. (United States)

Symposium Cochair

Donis G. Flagello, Nikon Corporation of America (United States)

Technical Organizing Committees

Executive Committee

John A. Allgair, SEMATECH Inc. (United States) and Advanced Micro Devices, Inc. (United States)

Mircea V. Dusa, ASML MaskTools, Inc. (United States)

Donis G. Flagello, Nikon Corporation of America (United States)

Clifford L. Henderson, Georgia Institute of Technology (United States)

Bruno La Fontaine, Advanced Micro Devices, Inc. (United States)

Harry J. Levinson, Advanced Micro Devices, Inc. (United States)

Christopher J. Progler, Photronics, Inc. (United States)

Christopher J. Raymond, Nanometrics, Inc. (United States)

Michael L. Rieger, Synopsys, Inc. (United States)

Franklin M. Schellenberg, Mentor Graphics Corporation (United States)

Vivek K. Singh, Intel Corporation (United States)

Advisory Committee

Robert D. Allen, IBM Almaden Research Center (United States)

William H. Arnold, ASML US Inc. (United States)

Timothy A. Brunner, IBM Thomas J. Watson Research Center (United States)

Ralph R. Dammel, AZ Electronic Materials USA Corporation (United States)

Roxann L. Engelstad, University of Wisconsin, Madison (United States)

Roderick R. Kunz, MIT Lincoln Laboratory (United States)

Harry J. Levinson, Advanced Micro Devices, Inc. (United States)

Burn Lin, Taiwan Semiconductor Manufacturing Company, Ltd. (Taiwan)

Chris A. Mack, LithoGuru.com (United States)

Victor Pol, Freescale Semiconductor, Inc. (United States) 
Michael T. Postek, National Institute of Standards and Technology (United States)

Luc Van den Hove, IMEC vzW (Belgium)

C. Grant Willson, The University of Texas at Austin (United States) Anthony Yen, Taiwan Semiconductor Manufacturing Company, Ltd. (Taiwan) 


\title{
Conference Committee
}

\author{
Conference Chair
}

Frank M. Schellenberg, Mentor Graphics Corporation (United States)

Conference Cochair

Bruno M. La Fontaine, Advanced Micro Devices, Inc. (United States)

Program Committee

David T. Attwood, Jr., Lawrence Berkley National Laboratory (United States) and University of California, Berkeley (United States)

James W. Blatchford, Texas Instruments Inc. (United States)

Kevin D. Cummings, ASML US, Inc. (United States)

Elizabeth A. Dobisz, Hitachi Global Storage Technologies (United States)

Benjamin G. Eynon, Jr., Molecular Imprints, Inc. (United States)

Michael Goldstein, SEMATECH Inc. (United States)

Francis Goodwin, SEMATECH Inc. (United States)

Timothy R. Groves, CNSE, SUNY, University at Albany (United States)

Cynthia Hanson, Space and Naval Warfare Systems Command (United States)

Daniel J. C. Herr, Semiconductor Research Corporation (United States)

Tatsuhiko Higashiki, Toshiba Corporation (Japan)

Frances A. Houle, IBM Almaden Research Center (United States)

Sung-Woo Lee, SAMSUNG Electronics Company, Ltd. (South Korea)

Michael J. Lercel, IBM Corporation (United States)

James A. Liddle, National Institute of Standards and Technology (United States)

Lloyd C. Litt, SEMATECH Inc. and Advanced Micro Devices, Inc. (United States)

Hans Loeschner, IMS Nanofabrication AG (Austria)

R. Scott Mackay, Mackay and Associates (United States)

Patrick P. Naulleau, Lawrence Berkeley National Laboratory (United

States)

Shinji Okazaki, Hitachi, Ltd. (Japan)

Laurent Pain, STMicroelectronics (France)

Jan Hendrik Peters, Advanced Mask Technology Center (Germany)

Douglas J. Resnick, Molecular Imprints, Inc. (United States)

Kazuaki Suzuki, Nikon Corporation (Japan)

William M. Tong, Hewlett-Packard Laboratory (United States)

Kevin T. Turner, University of Wisconsin, Madison (United States) 
Session Chairs

1 Keynotes: EUV

Bruno M. La Fontaine, Advanced Micro Devices, Inc. (United States)

Frank M. Schellenberg, Mentor Graphics Corporation (United States)

2 Keynotes: Alternative Litho Technologies

Frank M. Schellenberg, Mentor Graphics Corporation (United States)

Daniel J. C. Herr, Semiconductor Research Corporation (United States)

3 EUV Source

Francis Goodwin, SEMATECH Inc. (United States)

Bruno M. La Fontaine, Advanced Micro Devices, Inc. (United States)

$4 \quad$ EUV Mask

Kurt R. Kimmel, Advanced Mask Technology Center (Germany)

5 EBDW

Christof Klein, IMS Nanofabrication AG (Austria)

Cynthia Hanson, Space and Naval Warfare Systems Command (United States)

6 Multibeam and Tools Patterning

Timothy R. Groves, CNSE, SUNY, University at Albany (United States)

Laurent Pain, CEA, LETI (France)

$7 \quad$ EUV Printing

Shinji Okazaki, Hitachi, Ltd. (Japan)

Hans Meiling, ASML Netherlands B.V. (Netherlands)

8 EUV Lifetime

Andrea F. Wüest, SEMATECH Inc. (United States)

Kazuaki Suzuki, Nikon Corporation (Japan)

9 EUV Applications

Obert Wood II, Advanced Micro Devices, Inc. (United States)

10 EUV OPC

Patrick P. Naulleau, Lawrence Berkeley National Laboratory (United States)

11 Maskless

Tatsuhiko Higashiki, Toshiba Corporation (Japan)

Frank M. Schellenberg, Mentor Graphics Corporation (United States) 
Douglas J. Resnick, Molecular Imprints, Inc. (United States)

Lloyd C. Litt, Advanced Micro Devices, Inc. (United States)

13 Nanoimprint II

Benjamin G. Eynon, Jr., Molecular Imprints, Inc. (United States)

William M. Tong, Hewlett-Packard Laboratory (United States)

14 EUV Tools

Shinji Okazaki, Hitachi, Ltd. (Japan)

David T. Attwood, Jr., Lawrence Berkley National Laboratory (United States) and University of California, Berkeley (United States)

15 EUV Resist

Patrick P. Naulleau, Lawrence Berkeley National Laboratory (United States)

Thomas I. Wallow, Advanced Micro Devices, Inc. (United States)

16 Nanoimprint Materials

Frances A. Houle, IBM Almaden Research Center (United States)

Elizabeth A. Dobisz, Hitachi Global Storage Technologies, Inc. (United States)

17 Directed Self Assembly

Kevin T. Turner, University of Wisconsin, Madison (United States)

Steven L. Prins, Texas Instruments Inc. 
Downloaded From: https://www.spiedigitallibrary.org/conference-proceedings-of-spie on 25 Apr 2023

Terms of Use: https://www.spiedigitallibrary.org/terms-of-use 


\section{Introduction}

Welcome to the 2009 conference on Alternative Lithographic Technologies. I am very pleased to have been chair of this conference, and in this volume you will find papers describing some very exciting developments in several fields.

In previous years, this conference has gone by the name of "Emerging Lithographic Technologies." However, that is something of a misnomer; rarely has a technology "emerged" from this conference into mainstream CMOS patterning. This year, we have changed the name to better reflect the true nature of the conference, that of Alternative Lithography methods such as nanoimprint and EUV, as well as patterning for Alternative Technologies such as disk drives and photonics. We hope to provide a forum for both of these technology areas as the conference grows and evolves.

2009 was not the best year for the economy. With great uncertainty in world markets, and fabs running at half capacity, there is no doubt that times are troubled. In spite of that, I was very pleased to see that most of the conference sessions had their usual attendance, and some even increased over previous years. This shows a willingness to still pursue research, creativity, and innovation to address manufacturing problems, and gives great hope for the future of these promising technologies.

The most promising developments seen here were in the EUV Lithography community. Nearly 100 papers on EUV were presented, and tremendous progress has been made in all mission-critical areas (source power, optics contamination, resist efficacy). It remains to be seen, however, whether all aspects of the technology will come together to be reliable enough to provide a cost-effective solution for future technology nodes.

Outstanding work was also presented in nanoimprint and parallel e-beam technology. Nanoimprint has proven to be viable for creating patterned media for disk drives, and is on its way to production. And initial demonstrations for CMOS manufacturing have been explored both in the US and in Japan. In the meantime, recent progress from several suppliers of multiple-e-beam tools in the US, Europe and Japan show that this technology is taking the steps needed to demonstrate the capabilities needed for printing large areas.

And finally, we also have a set of papers on self-assembly materials, which spontaneously form patterns on the nano-scale, requiring patterning only on a larger, micro-scale to "direct" the formation of these smaller features.

I am very impressed by the strength and diversity of the work presented here; I hope you are as well. Of course, this would not be possible without the help of my 
Cochair, Bruno La Fontaine of AMD, the conference Program Committee, and of course, the SPIE staff. I thank them especially for their contributions in making this conference a success.

Frank M. Schellenberg 